\title{
Jia-Wei-Jiao-Tai-Wan ameliorates type 2 diabetes by improving $\beta$ cell function and reducing insulin resistance in diabetic rats
}

Guang Chen ${ }^{1 \dagger}$, Xueping Yang ${ }^{2 \dagger}$, Xiaoyu Yang ${ }^{3}$, Lingli Li ${ }^{4}$, Jinlong Luo ${ }^{5}$, Hui Dong ${ }^{2}$, Lijun Xu ${ }^{2}$, Ping Yi ${ }^{1}$, Kaifu Wang ${ }^{2}$, Xin Zou ${ }^{2}$ and Fuer Lu 2* $^{*}$

\begin{abstract}
Background: Jia-Wei-Jiao-Tai-Wan (JWJTW), composed of Jiao-Tai-Wan (Cinnamomum cassia and Rhizoma coptidis) and other antidiabetic herbs, including Astragalus membranaceus, Herba Gynostemmatis, Radix Puerariae Lobatae, Folium Mori and Semen Trigonellae, is widely used to treat diabetes and has demonstrated a curative effect in the clinic, but the potential mechanism is unknown. This study aimed to explore the effects of JWJTW on diabetic rats and to clarify the underlying mechanism.
\end{abstract}

Methods: JWJTW was prepared, and the main components contained in the formula were identified by highperformance liquid chromatography (HPLC) fingerprint analysis. Diabetic rats induced by streptozotocin (STZ) and a high-sucrose-high-fat diet were treated with two concentrations of JWJTW (1.025 and $2.05 \mathrm{~g} / \mathrm{kg} / \mathrm{d})$ for 100 days. The oral glucose tolerance test (OGTT), insulin release test (IRT) and insulin tolerance test (ITT) were performed to measure the glycometabolism of the diabetic rats at the end of the treatment period. Blood was collected to determine the serum lipid levels of the diabetic rats. Nitric oxide (NO), malondialdehyde (MDA), superoxide dismutase (SOD) and glutathione peroxidase (GSH-px) were detected in pancreas homogenates to analyze the oxidative stress in the pancreata of diabetic rats, and the expression levels of pancreatic and duodenal homeobox 1 (PDX-1) and insulin in the pancreas were tested by Western blot to measure pancreatic islet function. In addition, Western blots were used to measure the expression of proteins related to the insulin signaling pathway in skeletal muscle of the diabetic rats.

Results: The results showed that the administration of JWJTW could ameliorate impairments in glucose tolerance, insulin release function and insulin tolerance in diabetic rats. JWJTW could also dose-dependently reduce serum lipid levels in diabetic rats. JWJTW restrained oxidative stress by decreasing the expression of NO and MDA and increasing the expression of SOD and GSH-px. JWJTW improved the function of pancreatic $\beta$ cells by increasing PDX-1 and insulin expression. In addition, JWJTW restored the impaired insulin signaling; upregulated phospho-insulin receptor (plnsR) expression, insulin receptor substrate (IRS) tyrosine phosphorylation, phosphatidylinositol 3-kinase (PI3K) (p85), and glucose transporter 4 (GLUT4) expression; and downregulated the serine phosphorylation of IRS.

Conclusions: This study suggests that JWJTW can ameliorate type 2 diabetes by improving $\beta$ cell function and reducing insulin resistance in diabetic rats.

Keywords: Jia-Wei-Jiao-Tai-Wan (JWJTW), Type 2 diabetes mellitus, Pancreatic $\beta$ cell, Oxidative stress, Insulin resistance

* Correspondence: felu@tjh.tjmu.edu.cn

${ }^{\dagger}$ Equal contributors

${ }^{2}$ Institute of Integrative Traditional \& Western Medicine, Tongji Hospital,

Tongji Medical College, Huazhong University of Science \& Technology,

Wuhan 430030, China

Full list of author information is available at the end of the article 


\section{Background}

Diabetes mellitus is a continuously growing health problem that causes substantial morbidity, mortality and long-term complications. In 2015, the International Diabetes Federation estimated that, 415 million adults aged $20-70$ years worldwide had type 2 diabetes mellitus (T2DM); $75 \%$ of those affected lived in low-and middleincome countries, $46.5 \%$ had not been diagnosed and approximately 5 million people died of diabetes each year. By 2040, 642 million people are expected to suffer from diabetes [1]. Diabetes consumes approximately $12 \%$ of the world's medical costs (approximately $\$ 673$ billion), so there is a worldwide search for better pharmacologic agents to control T2DM.

T2DM is characterized by the dysregulation of carbohydrate, lipid and protein metabolism and it results from impaired insulin secretion, insulin resistance (IR) or a combination of both [2]. IR refers to a reduction in the efficiency by which insulin promotes glucose uptake and utilization by peripheral tissues, such as liver, muscle and adipose tissue. Under these circumstances, excessive insulin is secreted by body to maintain the stability of blood glucose, leading to hyperinsulinemia; thus, it is important to repair the insulin signaling pathway of peripheral tissue in the treatment of T2DM. Impaired insulin secretion is caused by the apoptosis of pancreatic $\beta$ cells, resulting in insufficient insulin production that can no longer meet physiological requirements. Thus, the achievement of durable glycemic control requires antidiabetic medications that can reverse the pathophysiological defects present in T2DM [3, 4], such as the apoptosis of pancreatic $\beta$ cells and peripheral tissue IR.

Diabetes has a long history in ancient China and other countries and more and more increasing numbers of herbs [5] have been widely used as medicines in the treatment of T2DM. Jiao-Tai-Wan, consisting of Rhizoma Coptidis and Cortex Cinnamomi cassiae, was first described by Han Yi (in the Chinese Ming Dynasty) in his treatise "Han Si Yi Tong". Jiang et al. demonstrated that Jiao-Tai-Wan could decrease blood lipid levels and increase insulin sensitivity in diabetic rats [6]. Zou et al. revealed the effects of Jiao-Tai-Wan on the deposition of fat in the pancreas and the apoptosis of pancreatic islet $\beta$ cells [7]. Our previous research also indicated an antihyperglycemic effect of Jiao-Tai-Wan in diabetic rats [8]. However, because the combination of two herbs is too simple to treat the complex symptoms of diabetic patients, the clinical application of Jiao-TaiWan has been limited. Jiao-Tai-Wan was improved by the addition of other antidiabetic herbs, including Astragalus membranaceus, Herba Gynostemmatis, Radix Puerariae Lobatae, Folium Mori and Semen Trigonellae, to form Jiao-Wei-Jiao-Tai-Wan (JWJTW). Sun et al. demonstrated that Astragalus membranaceus could inhibit the extrinsic and intrinsic apoptotic pathways in high-glucose-stimulated $\mathrm{H} 9 \mathrm{C} 2$ cells [9]. Gypenosides extracted from Herba Gynostemmatis also had a lipidlowering effect on nonalcoholic fatty liver disease (NAFLD) rats [10]. Researchers have revealed that puerarin, which is the main component of Radix Puerariae Lobatae, attenuates IR in 3T3L1 cells and diabetic rats [11-13]. Moreover, Folium Mori [14, 15] and Semen Trigonellae [16] also have antidiabetic effects on diabetic rats. JWJTW also had a great hypoglycemic effect in clinical T2DM treatment, and a patent has been issued (IPC Classification Nos. A61K36/185, A61P3/10, A61P3/ 06, A61P3/00 and A61K36/718). It was demonstrated that JWJTW could ameliorate oxidative stress and reduce the apoptosis of retinal ganglion cells in diabetic rats [17]. In addition, JWJTW was reported to reduce the fasting blood glucose (FBG) levels of T2DM patients in a clinical trial [18]. On the basis of these research studies and clinical applications of JWJTW to treat T2DM, a further animal experiment was conducted by us to analyze the potential mechanism of the antidiabetic action of JWJTW.

\section{Methods}

\section{Herbal materials and the preparation of JWJTW}

The medicinal plants used to prepare JWJTW are listed in Table 1. These plants were purchased from Hubei Herbal Materials Company (Wuhan, China). The voucher specimens were conserved at the herbal herbarium of Hubei University of Traditional Chinese Medicine, and were authenticated by Prof. Keli Chen at the School of Pharmacy, Hubei University of Chinese Medicine, based on their microscopic and macroscopic characteristics. JWJTW decoction was prepared in the Institute of Integrative Traditional Chinese and Western Medicine, Tongji Hospital, Tongji Medical College, Huazhong University of Science \& Technology (HUST, China). The preparation procedure (Fig. 1) was as

Table 1 Composition of Jia-Wei-Jiao-Tai-Wan (JWJTW)

\begin{tabular}{lll}
\hline Medicinal plant & Botanical plant name & Amount (g) \\
\hline Rhizoma coptidis & Coptis chinensis Franch & 3.0 \\
Cinnamomum cassia & Cinnamomum cassia Presl. & 1.5 \\
Radix Astragalus & $\begin{array}{l}\text { Astragalus membranaceus } \\
\text { (Fisch.) Bge. var. monholicus }\end{array}$ & 7.5 \\
& $\begin{array}{l}\text { (Bge.) Hsiao } \\
\text { Herba Gynostemmatis }\end{array}$ & $\begin{array}{l}\text { Gynostemma pentaphyllum } \\
\text { (Thunb.) Makino }\end{array}$ \\
Radix Puerariae Lobatae & Pueraria lobata (Willd.) Ohwi & 7.5 \\
Folium Mori & Morus alba L. & 6.0 \\
Semen Trigonellae & Trigonella foenum-graecum L. & 6.0 \\
\hline
\end{tabular}




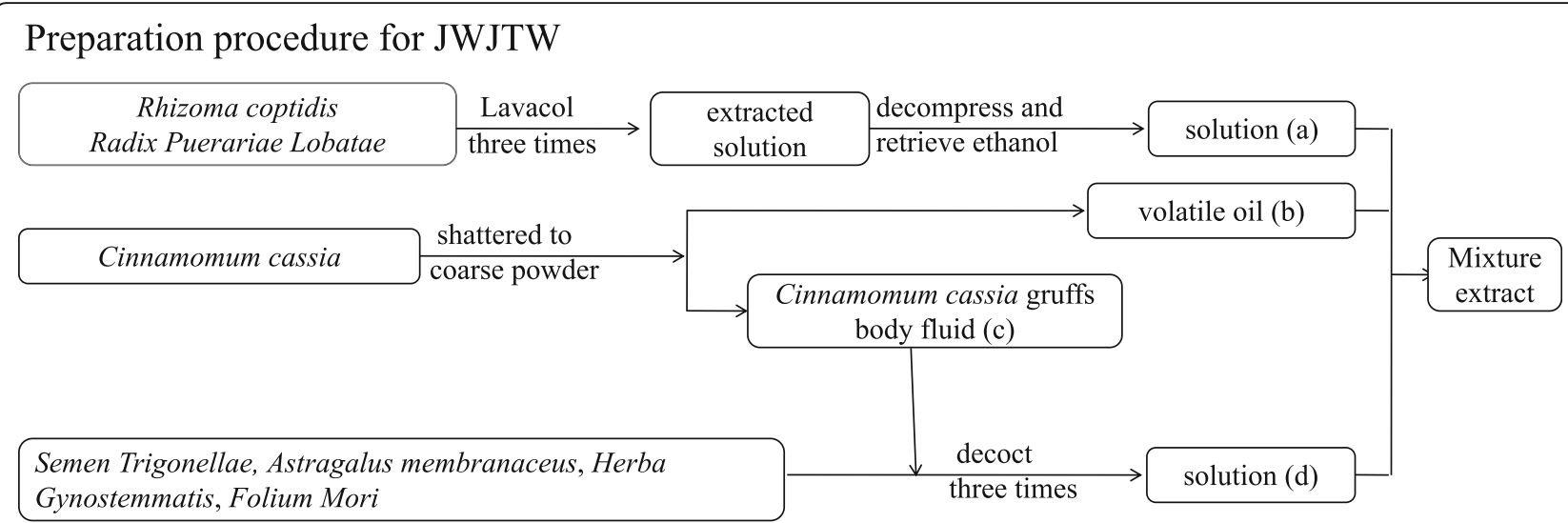

Fig. 1 Preparation procedure for JWJTW

follows: Rhizoma coptidis and Radix Puerariae Lobatae were extracted three times with Lavacol, and the extracting solutions were combined. The ethanol in the combined extracting solution was decompressed and retrieved as solution (a) with no ethanol flavor. Cinnamomum cassia was shattered to a coarse powder and then extracted as a volatile oil (b) with wet distillation. The Cinnamomum cassia gruff body fluid was reserved as (c). Semen Trigonellae, Astragalus membranaceus, Herba Gynostemmatis, Folium Mori and (c) were decocted three times after the addition of distilled water. The above solution (d) was concentrated with decompression. The concentrated extraction was added to extracts (a) and (b). The mixture was stored at $4{ }^{\circ} \mathrm{C}$ after vacuum dehydration.

\section{High-performance liquid chromatography (HPLC) fingerprint of the extract}

HPLC fingerprinting of the JWJTW extract was performed to identify the main chemical constituents in the samples. Trigonelline, puerarin, coptisine, jateorhizine, berberine, palmatine and cinnamic acid were used as standard substances. The extract was dissolved in water at a concentration of $1 \mathrm{~g} / \mathrm{ml}(\mathrm{w} / \mathrm{v})$ and then was diluted with methanol-water (50:50) to $0.5 \mathrm{~g} / \mathrm{ml}$ (w/v). HPLC analysis was performed using an Eclipse XDB-C18 column $(4.6 \times 250 \mathrm{~mm}, 5 \mu \mathrm{m}$, Aglient, USA $)$ eluted with mobile phases of methanol (A) and $0.1 \%$ phosphoric acid (B) with gradient elution (Table 2). The flow rate was $1.0 \mathrm{ml} / \mathrm{min}$ and the detection wavelength was $280 \mathrm{~nm}$.

Table 2 The mobile phases of HPLC fingerprint

\begin{tabular}{lll}
\hline Time (min) & Methanol (A) & $0.1 \%$ phosphoric acid (B) \\
\hline 0 & 5 & 95 \\
10 & 5 & 95 \\
110 & 55 & 45 \\
113 & 5 & 95 \\
120 & 5 & 95 \\
\hline
\end{tabular}

\section{Animals and treatment}

Healthy male Wistar rats aged 9 weeks (220-250 g) were purchased from Hubei Province Experimental Animal Research Center (grade specific pathogen-free). The animals were housed in an environmentally controlled breeding room (temperature: $20 \pm 2{ }^{\circ} \mathrm{C}$, humidity: $60 \pm$ $5 \%, 12-\mathrm{h}$ dark/light cycle). Water and food were given ad libitum. The animal studies were overseen and approved by the Animal Ethics Committee of Tongji Medical College, HUST before and during the experiment ([2015] IACUC Number: 305). After one-week of adaptive feeding, the rats were fasted for $12 \mathrm{~h}$ and were injected with streptozotocin (STZ, Sigma Chemical Company, St. Louis, Missouri) at a dose of $30 \mathrm{mg} / \mathrm{kg}$ through the tail vein. STZ was dissolved in sodium citrate saline buffer ( $\mathrm{pH}$ 4.5) and injected immediately within a few minutes to avoid degradation. Then, the rats were fed a high-sucrose-high-fat diet. Two weeks after the injection, impaired glucose tolerance (IGT) rats, as determined by an oral glucose tolerance test (OGTT), were randomly divided into three groups as follows: (1) $1.025 \mathrm{~g} / \mathrm{kg} / \mathrm{d}$ JWJTW group $(n=11)$, intragastric administration (ig) of $1.025 \mathrm{~g} / \mathrm{kg} / \mathrm{d}$ JWJTW; (2) $2.05 \mathrm{~g} / \mathrm{kg} / \mathrm{d}$ JWJTW group $(n=11)$, ig $2.05 \mathrm{~g} / \mathrm{kg} / \mathrm{d}$ JWJTW; and (3) diabetic group $(n=11)$, ig the vehicle for JWJTW. Another 11 rats established as a normal group received neither STZ injection nor drug administration. The high-sucrose-high-fat diet (sucrose: lard: milk powder: egg: conventional feed $=30: 40: 8: 4: 63$ ) was given to all the rats except for the normal group during the entire treatment period. Body weight was measured weekly to adjust the dose of JWJTW.

\section{Oral glucose tolerance test (OGTT), insulin release test (IRT) and insulin tolerance test (ITT)}

OGTT was performed 3 months after the first experimental treatment. Having been fasted for $12 \mathrm{~h}$, the rats were orally administered $2.2 \mathrm{~g} / \mathrm{kg}$ glucose. Then, blood 
samples were collected from the tail vein at 0 (just before the glucose load), 30, 60, 120 and $180 \mathrm{~min}$ (after the glucose load) for glucose and insulin assays. ITT was performed 1 week after OGTT. Baseline glucose levels were determined after a $4 \mathrm{~h}$ fast. Insulin ( 0.4 units $/ \mathrm{kg}$ ) was injected subcutaneously. Then, blood samples were collected from the tail vein at 0 (just before the insulin injection), 30, 60, 90 and $120 \mathrm{~min}$ (after the insulin injection) for the glucose assay.

\section{Tissue preparation}

The anesthetic drug sodium pentobarbital $(80 \mathrm{mg} / \mathrm{kg}$, Goodbio, Hubei, China) and all other necessary measures were used to reduce animal suffering during experimental procedures. After anesthesia, blood samples were collected from the internal canthus vein and sera were prepared and stored at $-80{ }^{\circ} \mathrm{C}$ for the determination of serum lipid levels. To assess the effect of JWJTW on insulin receptor activation in vivo, rats were anesthetized with sodium pentobarbital at the end of the 100-day treatment period. Then, insulin (8 units/ $\mathrm{kg}$ weight) or saline was injected into the vein of rats, as previously described [19]. Rats were sacrificed with carbon dioxide euthanasia approximately $4 \mathrm{~min}$ after the insulin injection. After the animal's death was confirmed the soleus muscles were separated, washed with cold phosphate buffer, and cut into 200-300 mg portions, which were then immediately frozen in liquid nitrogen and stored separately at $-80{ }^{\circ} \mathrm{C}$. The pancreas was cut in half after dissection, with one part immediately frozen in liquid nitrogen and stored at $-80{ }^{\circ} \mathrm{C}$ and the other fixed in $4 \%$ paraformaldehyde.

\section{Measurement of glucose and lipid metabolic parameters}

Blood glucose was estimated with a commercially available glucose kit (Beijing North Kangtai Clinical Reagent Co., Ltd., China) based on the glucose oxidase method. Lipid parameters such as total cholesterol (TC), triglyceride (TG), low-densitylipoprotein (LDL) and free fatty acids (FFA) were measured following the manufacturer's instructions (Wenzhou Dongou Biological Engineering Co., Ltd., China).

\section{Nitric oxide (NO), malondialdehyde (MDA), superoxide dismutase (SOD) and glutathione peroxidase (GSH-px) levels in pancreas homogenates}

The contents of NO, MDA, SOD and GSH-px in the pancreas were measured as follows. The pancreas was thawed, weighed and homogenized with $0.9 \%$ saline. Then, the homogenate was centrifuged $(12,000 \mathrm{~g}$, $15 \mathrm{~min}, 4{ }^{\circ} \mathrm{C}$ ) and the supernatant was immediately used for the assessment of NO, MDA, GSH-px and SOD. The NO, MDA, SOD and GSH-px levels were measured with commercial kits (Nanjing Jiancheng Biological Engineering Institute).

\section{Histological staining}

The general morphology of formalin-fixed paraffinembedded pancreatic tissue sections $(4 \mu \mathrm{m})$ was determined by hematoxylin and eosin staining.

\section{Antibodies and chemicals}

The antibodies used in this study were purchased from the following manufacturers: the antibody against pancreatic and duodenal homeobox 1 (PDX-1) was purchased from Chemi-con (USA); against insulin and against insulin receptor (InsR- $\beta$ ) from Abcam (UK); against insulin receptor substrate (IRS)-1 from Upstate (USA); against phospho-insulin receptor substrate (pIRS)-1(Ser307) and pIRS-1(Tyr612), phospho-insulin receptor (pInsR)- $\beta$ (Tyr1361), phosphatidylinositol 3kinase (PI3K) (p85) and glucose transporter 4 (GLUT4) from Cell Signaling Technology (CST, USA) and against GAPDH from Wuhan Gugeshengwu Technology Co., Ltd. (China). SDS-PAGE gel preparation, phenylmethanesulfonyl fluoride (PMSF), protease inhibitor cocktail, eosin, and hematoxylin were purchased from Wuhan Goodbio Technology Co., Ltd. (China).

\section{Western blot analysis}

The expression of proteins involved in the insulin signaling pathway in skeletal muscle and the expression of PDX-1 and insulin in the pancreas were measured by Western blot. The proteins were separated via $10-12 \%$ SDS-PAGE ( $80 \mathrm{~V}, 0.5 \mathrm{~h}$ and then $120 \mathrm{~V}, 1 \mathrm{~h})$ and transferred to a $0.22 \mu \mathrm{m}$ nitrocellulose membrane $(280 \mathrm{~A}$, $1 \mathrm{kDa} / \mathrm{min})$. The membranes were blocked with bovine serum albumin (BSA) powder in ultrapure water for $1 \mathrm{~h}$ at room temperature and incubated with primary antibodies overnight at $4{ }^{\circ} \mathrm{C}$. The next day, secondary antibodies were applied to the membranes and incubated for $1 \mathrm{~h}$ at room temperature following 3 rounds of interval washes with Tris-buffered-saline and Tween 20 (TBST, $10 \mathrm{~min}$ each). Bands were visualized with Odyssey Infrared Imaging (LI-COR Biosciences, USA) and were normalized against GAPDH using Image-Pro Plus software (Media Cybernetics, USA).

\section{Statistical analysis}

Statistical analyses were performed using SPSS 19.0 software (SPSS Software Products, C hicago, IL, USA). All results are expressed as the means \pm S.D. Significant differences among the groups were evaluated with a one-way analysis of variance (ANOVA) and Dunnett's ttest, and $p<0.05$ was considered significant. 


\section{Results}

\section{HPLC fingerprint of the extract}

HPLC fingerprint chromatograms of JWJTW are shown in Fig. 2. By comparison with both the retention times and UV spectra of the reference standards, seven compounds in the extract were well identified. The compounds were as follows: trigonelline (PubChem CID: 5570), puerarin (PubChem CID: 5,281,807), coptisine (PubChem CID: 72,322), jateorhizine (PubChem CID:72,323), berberine
(PubChem CID: 2353), palmatine (PubChem CID: 19,009) and cinnamic acid (PubChem CID:444,539).

\section{Effects of JWJTW on glucose tolerance, insulin release} and insulin tolerance in diabetic rats

After 100 days of treatment with JWJTW, the serum glucose levels at $0,30,60,120$ and $180 \mathrm{~min}$ in the diabetic group were higher than those in the normal group. The serum glucose levels at all of the above time points

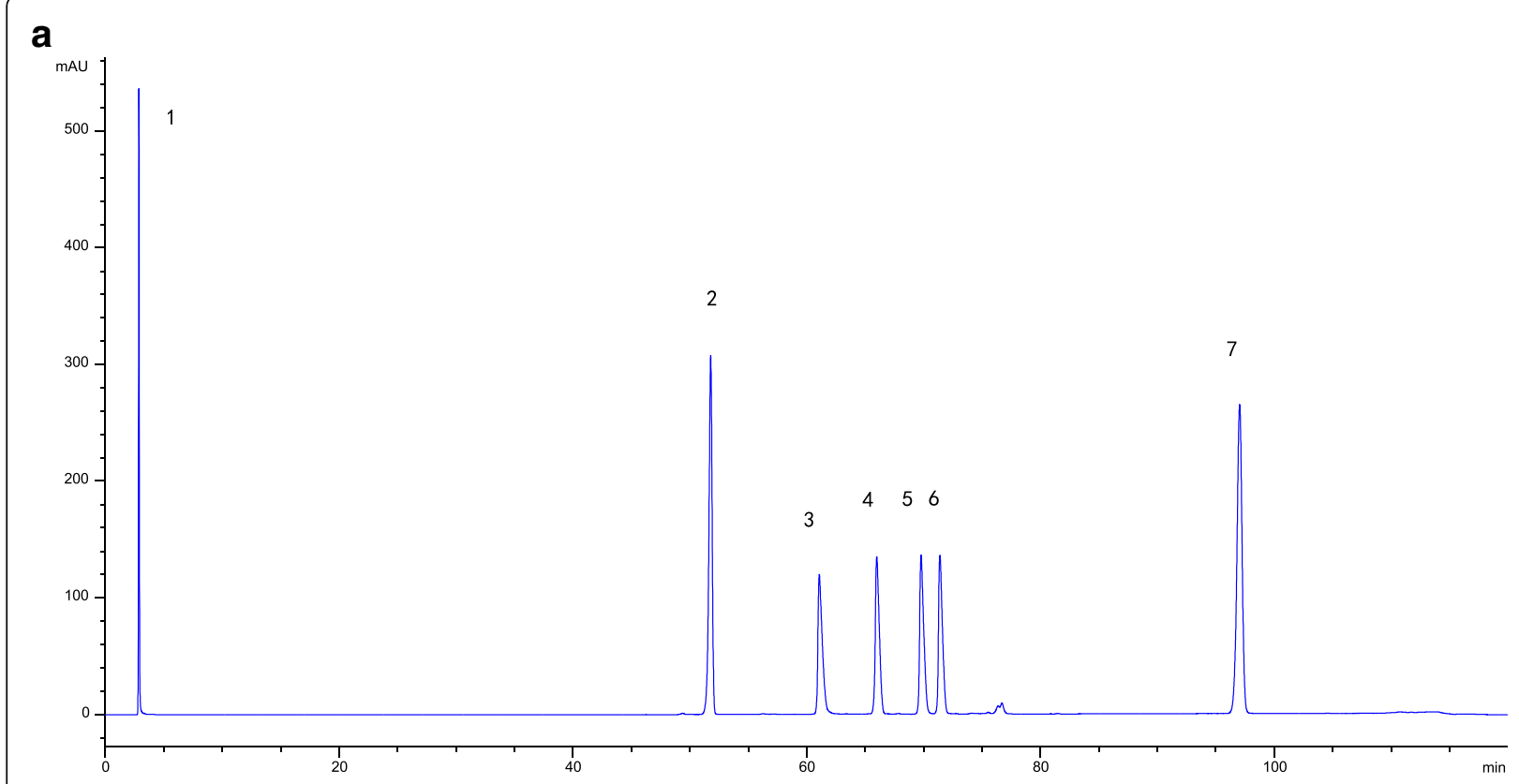

b

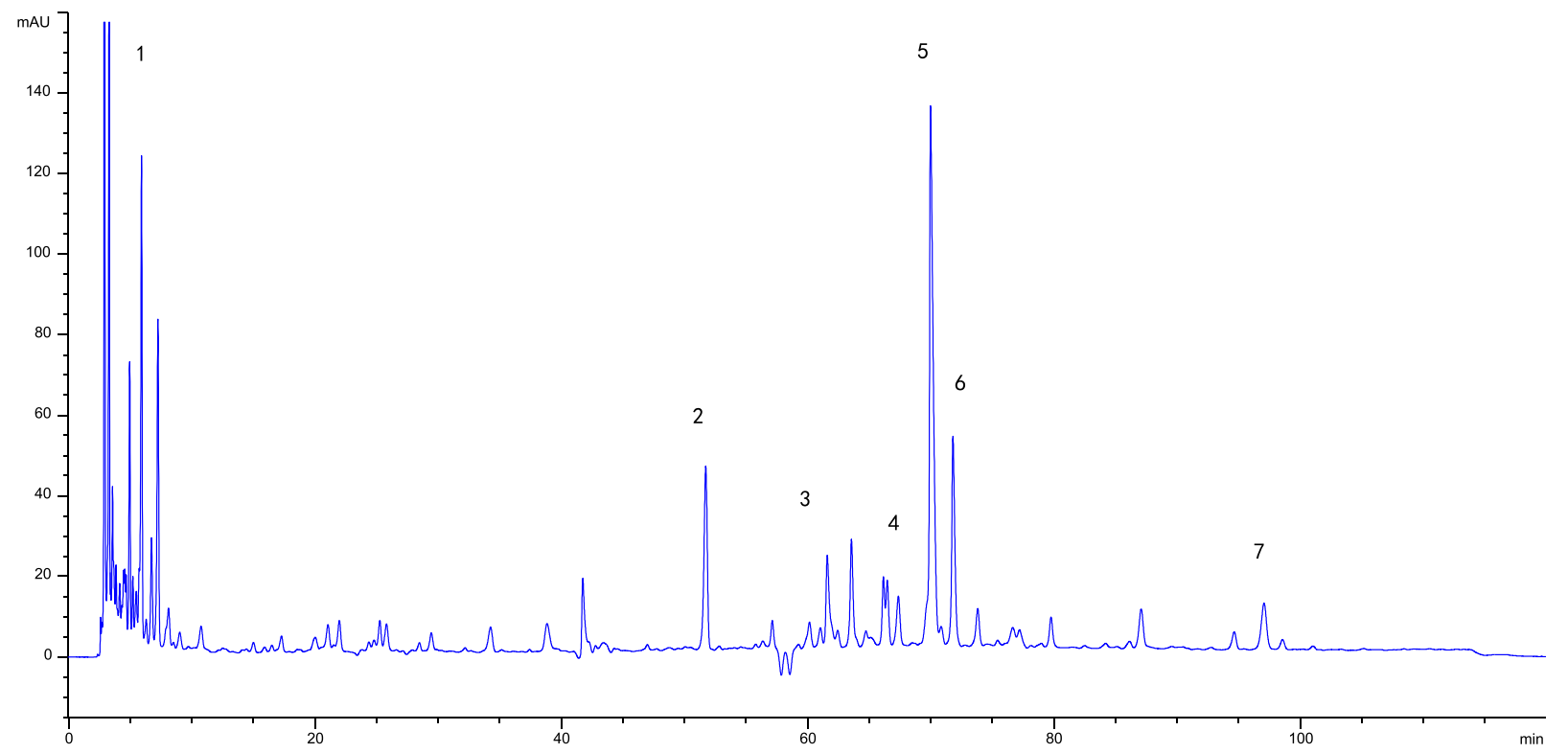

Fig. 2 HPLC fingerprint of the extract. HPLC fingerprint chromatograms of extracts of the reference standards (a) and JWJTW (b). In the chromatograms: (1) trigonelline (PubChem CID:5570); (2) puerarin (PubChem CID: 5,281,807); (3) coptisine (PubChem CID: 72,322); (4) jateorhizine (PubChem CID:72,323); (5) berberine (PubChem CID: 2353); (6) palmatine (PubChem CID: 19,009); (7) cinnamic acid (PubChem CID:444,539) 

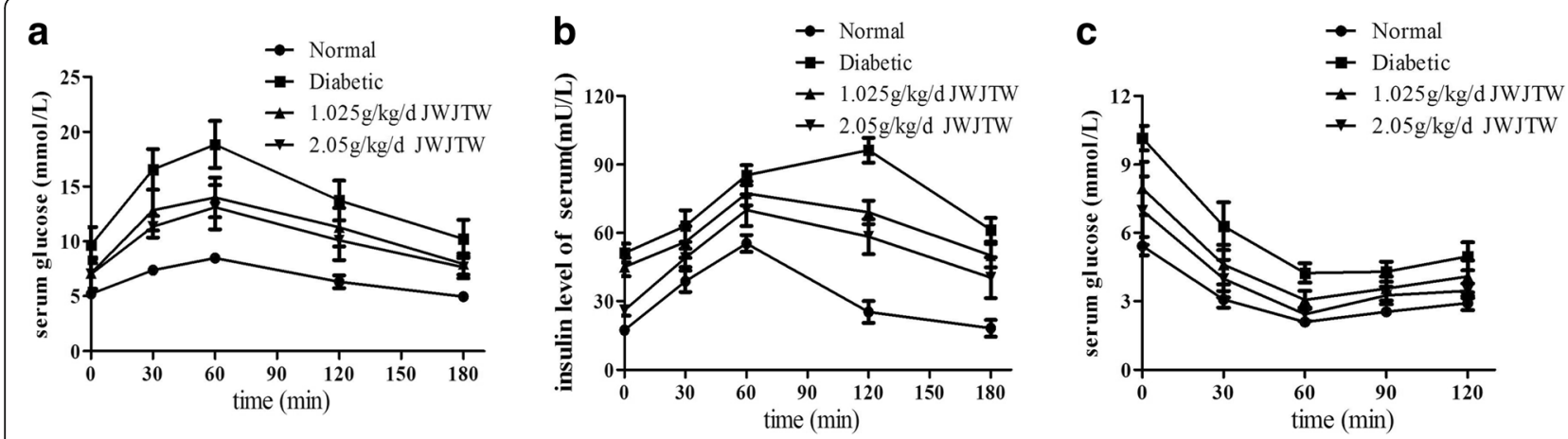

Fig. 3 Effects of JWJTW on glucose tolerance, insulin release and insulin tolerance in diabetic rats. a GTT of diabetic rats; $\mathbf{b}$ IRT of diabetic rats; $\mathbf{c}$ ITT of diabetic rats. Normal: normal group rats without streptozotocin (STZ) or high-sucrose-high-fat diet; Diabetic: diabetic rats induced by STZ and a high-sucrose-high-fat diet; $1.025 \mathrm{~g} / \mathrm{kg} / \mathrm{d}$ JWJTW: diabetic rats treated with $1.025 \mathrm{~g} / \mathrm{kg} / \mathrm{d}$ Jia-Wei-Jiao-Tai-Wan; $2.05 \mathrm{~g} / \mathrm{kg} / \mathrm{d}$ JWJTW: diabetic rats treated with $2.05 \mathrm{~g} / \mathrm{kg} / \mathrm{d}$ Jia-Wei-Jiao-Tai-Wan. GTT and IRT: Oral glucose tolerance test and insulin release test, respectively, performed on diabetic rats that were orally administered $2.2 \mathrm{~g}$ of glucose per $\mathrm{kg}$ after $12 \mathrm{~h}$ of fasting $(n=10)$. ITT: insulin tolerance test performed on diabetic rats injected with $0.4 \mathrm{U}$ insulin per $\mathrm{kg}$ after $4 \mathrm{~h}$ of fasting $(n=10)$

were lower in the JWJTW group than in the control group (Fig. 3a). As shown in Fig. 3b, diabetic group mice had higher serum insulin levels, but the release of insulin was delayed compared with the normal group; however, this was reversed with JWJTW treatment. The results of the ITT showed that significant IR had developed in the diabetic rats. Treatment with JWJTW dramatically decreased glucose levels at each point, indicating that insulin sensitivity had improved (Fig. 3c).

\section{Effects of JWJTW on lipid metabolic parameters of diabetic rats}

In rats administered STZ and then fed a high-sucrosehigh-fat diet for 100 days, TC,TG, LDL and FFA were all significantly elevated (Fig. 4), indicating that hyperlipidemia symptoms were also successfully established in the diabetic group. Daily treatment with JWJTW for 100 days dramatically decreased the TC, TG, LDL and FFA levels in serum $(" p<0.05, " p<0.01)$.

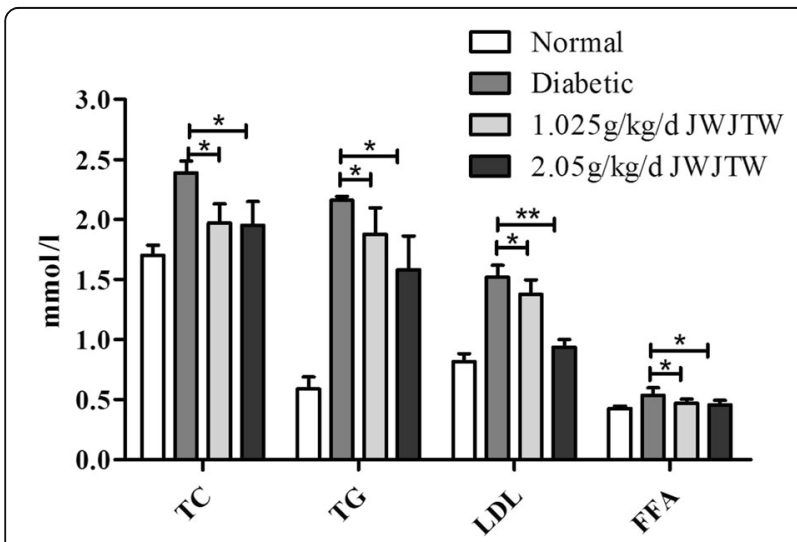

Fig. 4 Effects of JWJTW on lipid metabolic parameters of diabetic rats. TC: total cholesterol, TG: triglyceride, LDL: low-density lipoprotein, FFA: free fatty acids. ${ }^{*} p<0.05,{ }^{* *} p<0.01(n=10)$
Effects of JWJTW on the expression of oxidative stress in pancreas homogenates of diabetic rats

MDA, an important index for judging lipid peroxidation, was significantly increased in diabetic rats. After the treatment with JWJTW, the content of MDA in the pancreas was markedly decreased $(" p<0.05)$. Similar results were observed $\mathrm{NO}$, a type of reactive nitrogen species that can activate the oxidative stress mechanism (Fig. 5a, " $p<0.05, " m<0.01$ ). As shown in Fig. 5b, compared with the normal group, there were significant decreases in SOD and GSH-px in the diabetic group, and the trend was reversed in diabetic rats treated with JWJTW ( $p<0.05, " p<0.01)$.

\section{Effects of JWJTW on pancreatic islet number and structure of islets in diabetic rats}

The hematoxylin-eosin (HE) staining of pancreas tissue demonstrated that STZ injection and high-sucrose-highfat diet administration elicited severe injury to the pancreas. For example, the islet cell number decreased, and the diameter of pancreatic islets was diminished (Fig. 6a). Additionally, the structure of the pancreatic islets was disordered, vacuoles appeared, and nuclei were swollen (Fig. 6b). JWJTW administration produced a moderate expansion of islets, and pancreatic injury was significantly reduced.

\section{Effects of JWJTW on insulin and PDX-1 protein expression in pancreas of rats}

The Western blot analysis revealed that the expression of both PDX-1 and insulin in the pancreatic tissues was significantly decreased in diabetic rats compared with the normal group, while diabetic rats treated with JWJTW exhibited a greatly increased expression of PDX-1 and insulin (Fig. 7a, b " $p<0.05, " p<0.01$ ). 


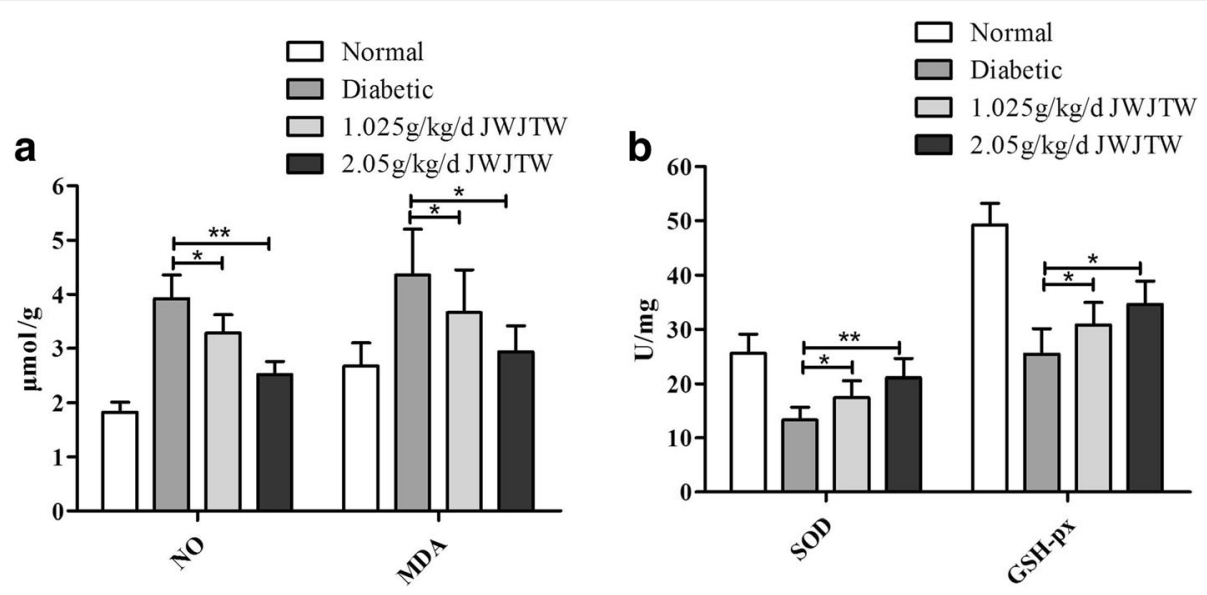

Fig. 5 Effects of JWJTW on SOD and GSH-px activity, MDA and NO content of pancreatic islets in diabetic rats. NO: nitric oxide, SOD: superoxide dismutase, GSH-px: glutathione peroxidase, MDA: malondialdehyde. a Homogenates of the pancreas were analyzed for NO and MDA. $\mathbf{b}$ Homogenates of the pancreas were analyzed for SOD and GSH-px. ${ }^{*} p<0.05,{ }^{* *} p<0.01(n=10)$

\section{Effects of JWJTW on the insulin signaling pathway in skeletal muscle of diabetic rats}

As shown in Fig. 8, there was no significant difference in insulin signaling pathway-related protein expression among the three groups without insulin stimulation; these proteins were all expressed at a low level. With insulin stimulation the degree of IR $\beta$ tyrosine phosphorylation (Fig. 8a, $" p<0.01$ ) in the skeletal muscle of the diabetic group rats was decreased significantly compared with that of the normal group rats but the 100-day treatment with JWJTW reversed this downward trend. The decrease in IRS-1 tyrosine phosphorylation levels in the muscle of diabetic rats was also ameliorated by JWJTW treatment, and IRS-1 serine (Ser307) phosphorylation in the same group was markedly downregulated with JWJTW treatment (Fig. 8b, " $p<0.05, " p<0.01$ ). Once phosphorylated IRS is activated, it combines with the PI3K regulatory subunit p85 and activates the downstream signaling pathway. After the 100-day JWJTW treatment, PI3K regulatory subunit p85 increased significantly in the skeletal muscles of the diabetic animals (Fig. 8c, $" p<0.05$ ). Moreover, the expression of GLUT4

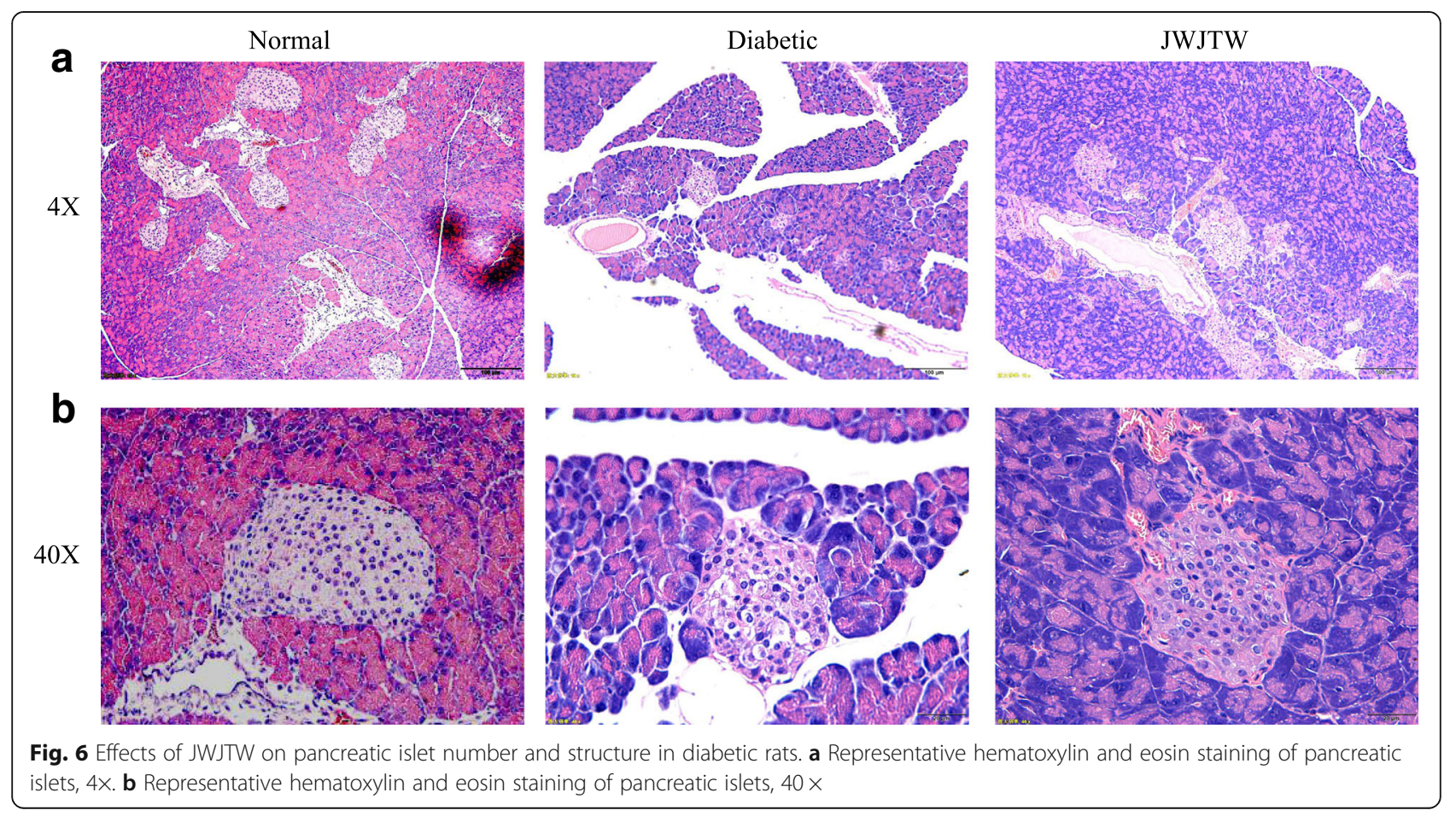




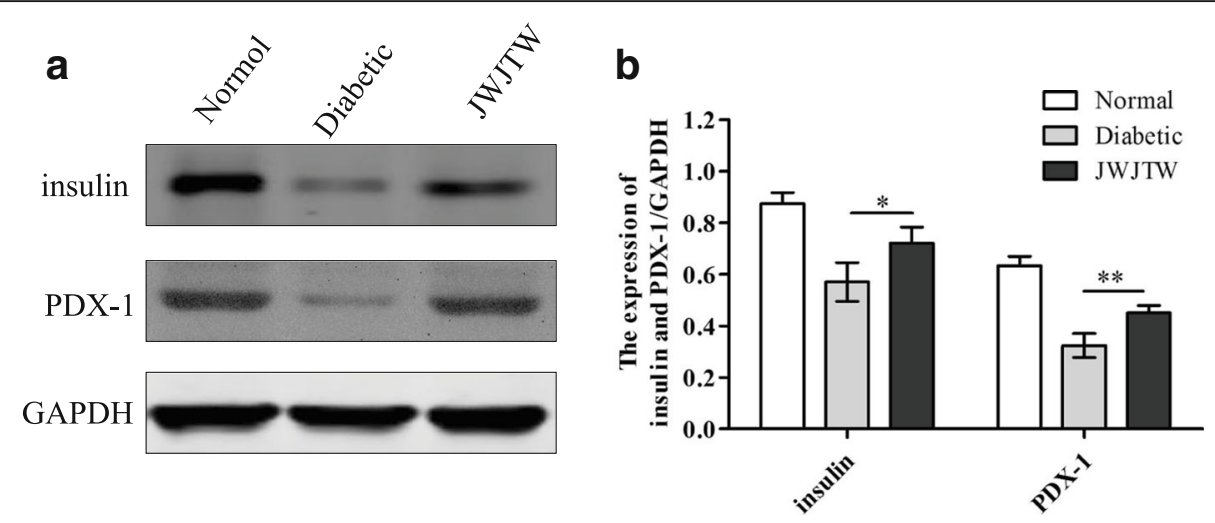

Fig. 7 Effects of JWJTW on insulin and PDX-1 protein expression in pancreatic islets of rats. a Western blot analysis of insulin and PDX-1 expression in pancreatic islets. $\mathbf{b}$ Bar graph of the signals for insulin and PDX-1. ${ }^{*} p<0.05,{ }^{* *} p<0.01(n=5)$

was increased by JWJTW (Fig. $8 \mathrm{~d}, \quad p<0.001$ ). Thus, the impaired insulin signaling pathway could be partly restored by JWJTW.

\section{Discussion}

Jiao-Tai-Wan is a traditional Chinese herbal formula that has been demonstrated to have antidiabetic effects in many animal experiments [20-22]. To address the complexity of clinical symptoms in diabetic patients, Astragalus membranaceus, Herba Gynostemmatis, Radix Puerariae Lobatae, Folium Mori and Semen Trigonellae were added to the Jiao-Tai-Wan prescription to yield JWJTW. Although JWJTW has achieved great antidiabetic effects in clinical applications and animal models, the specific mechanism is not clear. From the animal experiments in this study, we demonstrated that the prescription JWJTW consisting of the above mentioned drugs could ameliorate type 2 diabetes by improving $\beta$ cell function and reducing IR in rats.

Diabetic patients have impairments in glucose tolerance, insulin release function and insulin tolerance. This study found that JWJTW could ameliorate this abnormal condition in diabetic rats. It is now clear that IR is not solely a disorder of carbohydrate metabolism but also involves alterations in lipid metabolism [23]. It is well known that uncontrolled T2DM is accompanied by increased serum lipid levels. An encouraging result of this research was that 100-day treatment with JWJTW reduced the elevated levels of TC, TG, LDL and FFA in diabetic rats.

The disorder of glucose metabolism in diabetes is partially attributed to oxidative stress. Hyperglycemia leads to the over-production of free radicals, which can exert deleterious effects on the function of $\beta$ cells, rendering them susceptible to oxidative stress [24, 25]. Hyperglycemia can also degrade antioxidant enzyme defenses, thereby allowing reactive oxygen species (ROS) to damage cells and tissues. NO, a type of reactive nitrogen species that can activate oxidative stress, was reduced by JWJTW in this study. Moreover, the mechanism of impairing NO-mediated reactions is related to superoxide anions [26]; superoxide anions are metabolized to hydrogen peroxide by SOD, which is widely distributed throughout the body [27]. GSH, a strong cellular antioxidant present in many metabolic pathways [28], can reduce different oxidants after the addition of its hydrogen atom. In these reactions, two GSH molecules transform into one molecule of oxidized glutathione (GSSG). This reaction catalyzes the enzyme GSH-px [29] in cells. Our research also revealed that SOD and GSH-px activity in pancreas homogenates were both increased by JWJTW treatment. JWJTW also decreased the lipid peroxidation product, MDA in pancreas homogenates. In addition, researchers have revealed that ROS are closely related to the apoptosis of pancreatic $\beta$ cells [30], as is the morphology of the pancreatic islets [31]. Our research further revealed that JWJTW administration could partly repair the damaged structure of pancreatic islets caused by STZ and a high-sucrose-high-fat diet in diabetic rats.

It has been reported that ROS can not only induce the apoptosis of pancreatic $\beta$ cells but also decrease the expression of PDX-1 [32]. PDX-1 is regarded as a central transcription factor that regulates pancreatic development and islet $\beta$ cell function and maintains $\beta$ cell-specific gene expression [33]. This study demonstrated that the decreased expression of PDX-1 caused by STZ and a highsucrose-high-fat diet was upregulated by JWJTW treatment. It has been demonstrated that PDX-1 can regulate the expression of a lot of genes which are involved in maintaining beta-cell identity and function, such as insulin, glucokinase and inslet amyloid polypeptide [34]. In this research, JWJTW also improved insulin expression in the pancreas of diabetic rats by increasing PDX-1 expression.

IR is a general metabolic disorder that is attributable to the inefficient function of insulin in skeletal muscle, 


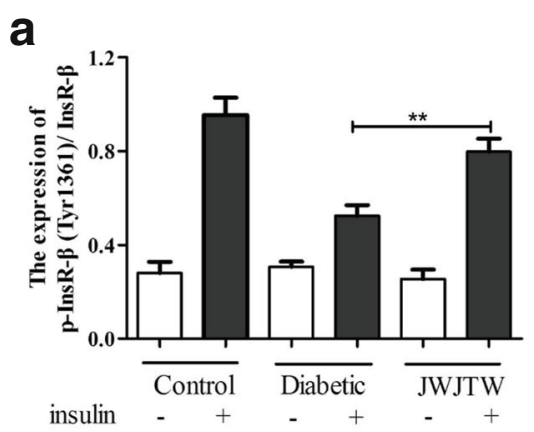

Phospho--InsR- $\beta^{\text {Tyr1361 }}$

$$
\text { InsR- } \beta
$$

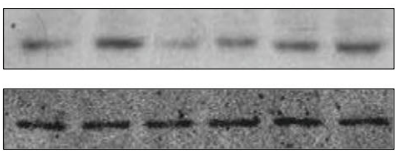

GAPDH

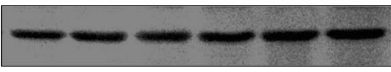

b
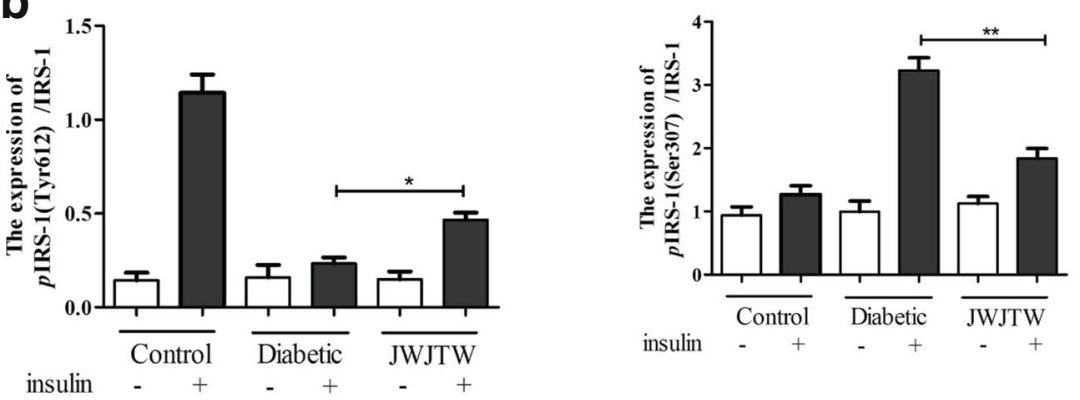

Phospho-IRS-1 Tyr612

Phospho-IRS-1 ${ }^{\text {Ser307 }}$

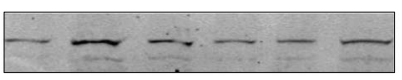

insulin

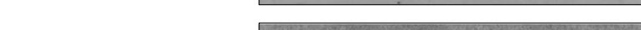

IRS-1

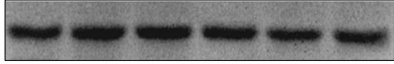

GAPDH

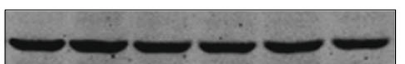

C

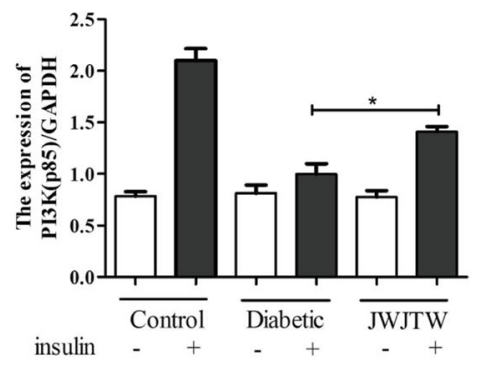

d

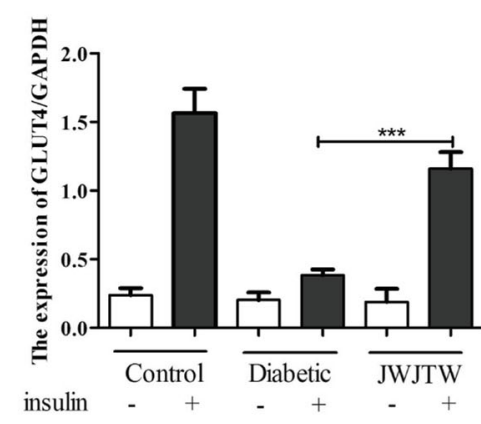

PI3K (p85)

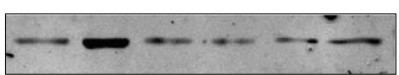

GLUT4

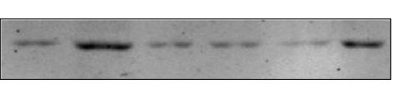

GAPDH

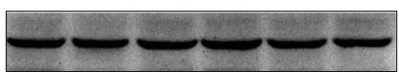

GAPDH

Fig. 8 Effect of JWJTW on insulin signaling in skeletal muscle of diabetic rats. Western blot analysis of phospho-InsR- $\beta^{\text {Tyr1361 }}$ (a), phospho-IRS- $1^{\text {Tyr612 }}$ and phospho-IRS-1 ${ }^{\text {Ser307 }}$ (b), PI3K (p85) (c) and GLUT4 (d). ${ }^{*} p<0.05,{ }^{* * *} p<0.01,{ }^{* * *} p<0.001(n=5)$ 
liver and/or adipose tissue. It has been documented that the effects of insulin on glucose uptake and metabolism are much greater in skeletal muscle than in liver and adipose tissue [35]. Defects in the insulin signaling cascade leading to impaired glucose utilization are believed to play a key role in the pathogenesis of IR [36]. Furthermore, studies have provided direct evidence of a complete biochemical pathway involving the insulin receptor, IRS-1, and PI3K that can account for important physiological actions by which insulin stimulates glucose uptake in muscle [37]. The present study demonstrated that STZ injection and a high-sucrose-high-fat diet caused reductions in the insulin receptor levels and the efficiency of its tyrosine phosphorylation in the muscle of rats. Our findings showed that JWJTW increased the level of IR $\beta$ tyrosine phosphorylation in the muscle of diabetic rats and suggest a beneficial role of JWJTW in the insulin signaling pathway.

Once activated, InsR binds to IRS- 1 and activates its phosphorylation. Serine phosphorylation of IRS-1 has been proposed as a general mechanism for the functional inhibition of the IRS-1 protein, and Ser307 phosphorylation is recognized as a molecular indicator of IR [38]. Tyrosine phosphorylation of IRS-1 leads to the binding of PI3K [38] and the activation of its enzymatic activity, a necessary step for the translocation of GLUT4 to the plasma membrane, which consequently elevates the rate of cellular glucose uptake in response to insulin [39].Our results showed that JWJTW treatment significantly inhibited the upregulation of phospho-IRS1 (Ser307) and the downregulation of IRS-1 tyrosine phosphorylation in the muscle of diabetic rats induced by STZ and a high-sucrose-high-fat diet. PI3K, which is downstream of IRS, plays a pivotal role in the insulin signaling pathway and was also increased by JWJTW. Our data also showed that JWJTW treatment achieved a definite improvement in the decreased GLUT4 expression in the skeletal muscle of diabetic rats.

\section{Conclusions}

This study confirmed that JWJTW can ameliorate type 2 diabetes by improving $\beta$ cell function and reducing IR in diabetic rats. Our results provide a further reference for the elucidation of the specific mechanisms underlying the hypoglycemic effect of JWJTW, and they also provide evidence for the clinical use of JWJTW to treat type 2 diabetes.

\footnotetext{
Abbreviations

BSA: Bovine serum albumin; FBG: Fasting blood glucose; FFA: Free fatty acids; GLUT4: Glucose transporter 4; GSH-px: Glutathione peroxidase; HE: Hematoxylin-eosin; HPLC: High-performance liquid chromatography; HUST: Huazhong University of Science \& Technology; ig: Intragastric Administration; IGT: Impaired glucose tolerance; InsR: Insulin receptor; IR: Insulin resistance; IRS: Insulin receptor substrate; IRT: Insulin release test; ITT: Insulin tolerance test; JWJTW: Jia-Wei-Jiao-Tai-Wan; LDL: Low-density
}

lipoprotein; MDA: Malondialdehyde; NAFLD: Nonalcoholic fatty liver disease; NO: Nitric oxide; OGTT: Oral glucose tolerance test; PDX-1: Pancreatic and duodenal homeobox 1; PI3K: Phosphatidylino-sitol3-kinase; plnsR: Phosphoinsulin receptor; pIRS: Phospho-insulin receptor substrate;

PMSF: Phenylmethanesulfonyl fluoride; ROS: Reactive oxygen species; SOD: Superoxide dismutase; STZ: Streptozotocin; T2DM: Type 2 diabetes mellitus; TBST: Tris-buffered-saline and Tween 20; TC: Total cholesterol; TG: Triglyceride

\section{Acknowledgements}

Not applicable.

\section{Funding}

This work was supported by the National Natural Science Foundation of China (No. 81673928, 81403254 and 81373871).

\section{Availability of data and materials}

The datasets used and/or analyzed during the current study are available from the corresponding author on reasonable request.

\section{Authors' contributions}

LF and CG conceived and designed the study. CG, YX (YXP), YX (YXY), LL and $X L$ performed the experiments. $C G, L L, Y X(Y X P)$ and $D H$ wrote the paper. $L$, YP, WK and ZX reviewed and edited the manuscript. All authors read and approved the manuscript.

\section{Ethics approval}

All procedures were overseen and approved by the Animal Ethics

Committee of Tongji Medical College, Huazhong University of Science \&

Technology before and during the experiment ([2015] IACUC Number: 305).

\section{Consent for publication}

Not applicable.

\section{Competing interests}

The authors declare that they have no competing interests.

\section{Publisher's Note}

Springer Nature remains neutral with regard to jurisdictional claims in published maps and institutional affiliations.

\section{Author details}

${ }^{1}$ Department of Integrative Traditional \& Western Medicine, Tongji Hospital, Tongji Medical College, Huazhong University of Science \& Technology, Wuhan 430030, China. ${ }^{2}$ Institute of Integrative Traditional \& Western Medicine, Tongji Hospital, Tongji Medical College, Huazhong University of Science \& Technology, Wuhan 430030, China. ${ }^{3}$ Department of Oncology, Xiangyang No. 1 Hospital, Xiangyang 441000, China. ${ }^{4}$ Department of Traditional Chinese Medicine, Pu'ai Hospital, Tongji Medical College, Huazhong University of Science \& Technology, Wuhan 430033, China. ${ }^{5}$ Department of Emergency, Tongji Hospital, Tongji Medical College, Huazhong University of Science \& Technology, Wuhan 430030, China.

Received: 30 June 2017 Accepted: 19 November 2017

Published online: 29 November 2017

References

1. Campos J, Ramos A, Szego T, Zilberstein B, Feitosa H, Cohen R. The role of metabolic surgery for patients with obesity grade i and type 2 diabetes not controlled clinically. Arq Bras Cir Dig. 2016;29(Suppl 1):102-6.

2. DeFronzo RA. FerranniniE, Groop L, Henry RR, Herman WH, Holst JJ, Hu FB, Kahn CR, Raz I, Shulman Gl, Simonson DC, Testa M a, ram Weiss. Type 2 diabetes mellitus. Nat Reviews Disease Primers. 2015;1:1-22.

3. De Fronzo RA. Banting lecture. From the triumvirate to the ominous octet: a new paradigm for the treatment of type 2 diabetes mellitus. Diabetes. 2009;58:773-95.

4. Abdul-Ghani MA, Puckett C, Triplitt C, Maggs D, Adams J, Cersosimo E, DeFronzo RA. Initial combination therapy with metformin, pioglitazone and exenatide is more effective than sequential add-on therapy in subjects with new-onset diabetes.Results from the efficacy and durability of initial combination therapy for type 2 diabetes (EDICT): a randomized trial. Diabetes Obes Metab. 2015;17:268-75. 
5. Islam MA, Akhtar MA, Khan MR, Hossain MS, Alam AH, Ibne-Wahed MI, Amran MS, Rahman BM, Ahmed M. Oral glucose tolerance test (OGTT) in normal control and glucose induced hyperglycemic rats with Coccinia cordifolia L. and Catharanthus roseus L. Pak J Pharm Sci. 2009:22:402-4.

6. Jiang ZS, Zhang SL, Yan H, Zhang XY. HeY. Effect of Jiaotai decoction onthe level of blood lipid and the sensitivity of insulin in rats with type 2 diabetes. Chinese journal of information on traditional. Chin Med. 2001;8:36-7.

7. Zou X, Liu DL, FE L, Dong H, L X, Luo YH, Wang KF. Effect of Jiao-tai-wan on the deposition of fat in pancreas and apoptosis of pancreatic $\beta$ cells. China J Chinese Materia Medica. 2014;39:2106-11.

8. Chen G, Lu F, Xu L, Dong H, Yi P, Wang F, Huang Z, Zou X. The anti-diabetic effects and pharmacokinetic profiles of berberine in micetreated with Jiaotai-wan and its compatibility. Phytomedicine. 2013;20:780-6.

9. Sun S, Yang S, Dai M, Jia X, Wang Q, Zhang Z, Mao Y. The effect of Astragalus polysaccharides on attenuation of diabetic cardiomyopathy through inhibiting the extrinsic and intrinsic apoptotic pathways in high glucose-stimulated $\mathrm{H} 9 \mathrm{C} 2$ cells. BMC Complement Altern Med. 2017;17(1):310.

10. Gou SH, Huang HF, Chen XY, Liu J, He M, Ma YY, Zhao XN, Zhang Y, Ni JM. Lipid-lowering, hepatoprotective, and atheroprotective effects of the mixture Hong-Qu and gypenosides in hyperlipidemia with NAFLD rats. J Chin Med Assoc. 2016;79(3):111-21.

11. Zhao $Y$, Zhou Y. Puerarin improve insulin resistance of adipocyte through activating Cb1 binding protein path. Chin J Integr Med. 2012;18(4):293-8.

12. Huang F, Liu K, Du H, Kou J, Liu B. Puerarin attenuates endothelial insulin resistance through inhibition of inflammatory response in an IKKB/IRS-1dependent manner. Biochimie. 2012;94(5):1143-50.

13. Zhang W, Liu CQ, Wang PW, Sun SY, WJ S, Zhang HJ, Li XJ, Yang SY. Puerarin improves insulin resistance and modulates adipokine expression in rats fed a high-fat diet. Eur J Pharmacol. 2010;649:398-402.

14. Riche DM, Riche KD, East HE, Barrett EK, May WL. Impact of mulberry leaf extract on type 2 diabetes (Mul-DM): a randomized, placebo-controlled pilot study. Complement Ther Med. 2017;32:105-8.

15. Liu CG, Ma YP, Zhang XJ. Effects of mulberry leaf polysaccharide on oxidative stress in pancreatic $\beta$-cells of type 2 diabetic rats. Eur Rev Med Pharmacol Sci. 2017;21(10):2482-8.

16. Pradeep SR, Srinivasan K.Amelioration of oxidative stress bydietary fenugreek (Trigonellafoenum-graecum L.) seeds is potentiated by onion (Allium cepa L.) in streptozotocin-induced diabetic rats. Appl Physiol Nutr Metab 2017:20:1-13.

17. Chen G, Yang HJ, Dong $H, X u L J$, Zou X, Huang ZY, Wang KF, Lu FE. Preventive and therapeutic effect of Jiawei Jiaotai pill onDiabetic retinopathy in diabetic rats and its potential mechanism. Chin J Experimental Traditional Med Formulae. 2013;19:183-7.

18. Quan SJ, Hei SY, Qian LL. Clinical observation of 70 cases of type 2 diabetes mellitus complicated with insomnia treated with Jia-Wei-Jiao-tai-wan. JNew Chin Med. 2012;44:85-6.

19. Tremblay F, Lavigne $C$, Jacques $H$, Marette A. Dietary cod protein restores insulin-induced activation of phosphatidylinositol 3-kinase/Akt and GLUT4 translocation to the T-tubules in skeletal muscle of high-fat-fed obese rats. Diabetes. 2003;52:29-37.

20. Zou X, Huang W, Lu F, Fang K, Wang D, Zhao S, Jia J, Xu L, Wang K, Wang $\mathrm{N}$, Dong $\mathrm{H}$. The effects of Jiao-tai-wan on sleep, inflammation and insulin resistance in obesity-resistant rats with chronic partial sleep deprivation. BMC Complement Altern Med. 2017;17(1):165.

21. Huang Z, Xu X, Lu F, Wang N, Chen G, Zhao Y, Zou X, Wang K, Dong H, Xu L. Jiao-Tai-Wan attenuates hepatic lipid accumulation in type 2 diabetes mellitus. Evid Based Complement Alternat Med. 2013;2013:567045.

22. Yuan L, Li HJ, Hu N, Tang P, Lin M, Zhong XY, Lu X. Study on hypoglycemic effect of Jiao-tai-wan with different proportions and its related mechanism. Chin J Exp Traditional Med Formulae. 2017;23:130-7.

23. Wang X, Zhou L, Li G, Luo T, Gu Y, Qian L, Fu X, Li F, Li J, Luo M. Palmitate activates AMP activated protein kinase and regulates insulin secretionfrom beta cells. Biochem Biophys Res Commun. 2007;352:463-8.

24. Brownlee M, Vlassara H, Cerami A. Non-enzymatic glycosylationand the pathogenesis of diabetic complications. Ann Intern Med. 1984;101:527-37.

25. Njoroge FG, Monnier VM. The chemistry of the Maillard reactionunder physiological conditions: a review. Prog ClinBiol Res. 1989;304:85-107.

26. Vallejo S, Angulo J, Peiro C, Nevado J, Sanchez-Ferrer A, Petidier R, Sanchez-Ferrer CF, Rodriguez-Manas L. Highly glycatedoxyhaemoglobin impairs nitric oxide relaxations in human mesentericmicrovessels. Diabetologia. 2000;43:83-90.
27. McCord JM, Fridovich I. Superoxide dismutase. An enzymic function for erythrocuprein (hemocuprein). J Biol Chem 1969;244: 6049-6055.

28. Robertson RP. Chronic oxidative stress as a central mechanism for glucose toxicity in pancreatic islet beta cells in diabetes. J Biol Chem. 2004;279: 42351-4.

29. Reiter RJ. Oxidative processes and antioxidative defense mechanismsin the aging brain. FASEB J. 1995;9:526-33.

30. Fu J, Cui Q, Yang B, Hou Y, Wang H, Xu Y, Wang D, Zhang Q, Pi J. The impairment of glucose-stimulated insulin secretion in pancreatic-cells caused by prolonged glucotoxicity and lipotoxicity is associated with elevated adaptive antioxidant response. Food Chem Toxicol. 2017;100:161-7.

31. Nurdiana S, Goh YM, Ahmad H, Dom SM, Syimal'ain Azmi N, Noor Mohamad Zin NS, Ebrahimi M. Changes in pancreatic histology, insulin secretion and oxidative status in diabetic rats following treatment with Ficus Deltoidea and vitexin. BMC Complement Altern Med. 2017;17(1):290.

32. Kaneto H, Kajimoto Y, Miyagawa J, Matsuoka T, Fujitani Y, Umayahara Y, Hanafusa T, Matsuzawa Y, Yamasaki Y, Hori M. Beneficial effects of antioxidants in diabetes : possible protection of pancreati c beta- cells against glucose toxicity. Diabetes. 1999;48(12):2398-406.

33. Kaneto H, Miyatsuka T, Shiraiwa T, Yamamoto K, Kato K, Fujitani $Y$ Matsuoka TA. Crucial role of PDX-1 in pancreas development,beta-cell differentiation, and induction of surrogate beta-cells. Curr Med Chem. 2007;14:1745-52.

34. McKinnon C, Docherty K. Pancreatic duodenal homeobox-1, PDX-1, a major regulator of beta cell identityand function. Diabetologia. 2001;44:1203-14.

35. Song XM, Ryder JW, Kawano Y, Chibalin AV, Krook A, Zierath JR. Muscle fiber type specificity in insulin signal transduction. Am J Phys. 1999;277:1690-6.

36. Shulman Gl. Cellular mechanisms of insulin resistance. J Clin Invest. 2000; 106:171-6.

37. Carvalho E, Rondinone C, Smith U. Insulin resistance in fat cells from obese Zucker ratsevidencefor an impaired activation and translocation of protein kinase B and glucose transporter4. Mol Cell Biochem. 2000;206:7-16.

38. Hirosumi J, Tuncman G, Chang L, Gorgun CZ, Uysal KT, Maeda K, Karin M, Hotamisligil GS. A central role for JNK in obesity and insulin resistance. Nature. 2002;420:333-6.

39. White MF. The IRS-signalling system: a network ofdocking proteins that mediate insulin action. Mol Cell Biochem. 1998;182:3-11.

\section{Submit your next manuscript to BioMed Central and we will help you at every step:}

- We accept pre-submission inquiries

- Our selector tool helps you to find the most relevant journal

- We provide round the clock customer support

- Convenient online submission

- Thorough peer review

- Inclusion in PubMed and all major indexing services

- Maximum visibility for your research

Submit your manuscript at www.biomedcentral.com/submit
Biomed Central 